BMJ Open

Diabetes

Research

\& Care

\section{Association of diabetic retinopathy with both sarcopenia and muscle quality in patients with type 2 diabetes: a cross- sectional study}

To cite: Fukuda T, Bouchi R, Takeuchi T, et al. Association of diabetic retinopathy with both sarcopenia and muscle quality in patients with type 2 diabetes: a cross-sectional study. BMJ Open Diab Res Care 2017;5:e000404. doi:10.1136/ bmjdrc-2017-000404

Received 21 February 2017 Revised 29 March 2017 Accepted 26 April 2017

\section{(a) CrossMark}

${ }^{1}$ Department of Molecular Endocrinology and Metabolism, Graduate School of Medical and Dental Sciences, Tokyo Medical and Dental University, Tokyo, Japan

${ }^{2}$ Center for Medical Welfare and Liaison Services, Tokyo Medical and Dental University, Tokyo, Japan

${ }^{3}$ Department of Preemptive Medicine and Metabolism, Graduate School of Medical and Dental Sciences, Tokyo Medical and Dental University, Tokyo, Japan

${ }^{4}$ CREST, Japan Agency for Medical Research and Development, Tokyo, Japan

Correspondence to Dr. Ryotaro Bouchi; bouchi.mem@tmd.ac.jp

\section{ABSTRACT}

Objective To examine whether the existence and severity of diabetic retinopathy (DR) could be associated with the prevalent sarcopenia and muscle quality in patients with type 2 diabetes.

Research design and methods This is a cross-sectional study of 316 patients with type 2 diabetes (mean age $65 \pm 12$ years; $38 \%$ female). Body compositions were measured by the dual-energy $X$-ray absorptiometry. Patients were divided into three groups: patients without DR (NDR), with non-proliferative DR (NPDR) and proliferative DR (PDR). Sarcopenia was diagnosed according to the criteria for Asians, using both skeletal muscle index (SMI) and grip strength $(\mathrm{kg})$. Muscle quality was also determined by the grip strength divided by SMI. Logistic regression analyses were carried out to assess the cross-sectional association of the severity of DR with sarcopenia. In addition, linear regression analyses were performed to determine the associations between DR and muscle quality. Selection of covariates in the multivariate logistic and linear regression analyses was done by a stepwise procedure.

Results Among the patients examined, NDR, NPDR and PDR were diagnosed in 261, 38 and 17 patients, respectively. The prevalence of sarcopenia significantly increased along with the progression of DR. Multivariate logistic regression analysis showed that PDR is significantly associated with sarcopenia (OR $7.78,95 \% \mathrm{Cl}$ 1.52 to $39.81, p=0.014$ ) and low muscle strength ( $O R$ $6.25,95 \% \mathrm{Cl} 1.15$ to $33.96, p=0.034$ ). Multivariate linear regression analysis additionally showed that the existence of DR was significantly associated with the muscle quality (standardized $\beta-0.136, p=0.005$ for NPDR, standardized $\beta$ $-0.146, p=0.003$ for PDR).

Conclusions This study provides evidence that PDR is significantly associated with sarcopenia, and the existence of DR increases the risk for low muscle quality in patients with type 2 diabetes.

\section{INTRODUCTION}

Diabetic retinopathy (DR) is one of the common microvascular complications in patients with diabetes and a leading cause of the visual impairment and blindness. ${ }^{1-3}$ DR

\section{Significance of the study}

What is already known about this subject?

- Several recent studies have reported that diabetes contributes to the burden of sarcopenia and diabetic microvascular complications are associated with sarcopenia. However, the association between diabetic retinopathy and sarcopenia remains largely unknown.

What are the new findings?

- In this study, the prevalence of sarcopenia significantly was increased along with the progression of diabetic retinopathy in patients with type 2 diabetes. In the multivariate models, proliferative diabetic retinopathy was significantly associated with sarcopenia, and the existence of diabetic retinopathy was significantly associated with the low muscle quality.

How might these results change the focus of research or clinical practice?

- Patients with diabetic retinopathy may be regarded as a high-risk group for sarcopenia and low muscle quality.

is associated with an increased risk for albuminuria, cardiovascular events, and all-cause mortality in patients with diabetes. ${ }^{45}$

Sarcopenia is defined as the loss of skeletal muscle mass and strength with aging and contributes to both physical disability and mobility limitations. ${ }^{67}$ In addition to aging, many chronic diseases are also associated with sarcopenia. ${ }^{8}$ Of the chronic disease, diabetes has been reported to be one of the significant contributors to the exacerbation of sarcopenia. ${ }^{9}$ The Health, Aging, and Body Composition (ABC) Study revealed that diabetes is associated with the rapid loss of skeletal muscle strength and mass. ${ }^{10}$ Interestingly, this study also demonstrated 
that muscle quality, defined as muscle strength standardized for muscle mass, declined more rapidly in patients with diabetes than in subjects without diabetes. Similarly, Volpato et al reported in a cross-sectional analysis of 835 participants that patients with diabetes have a lower muscle strength in lower extremities, despite having a larger muscle mass, and diabetes might cause mobility limitations via impaired muscle quality. ${ }^{11}$ In addition, either longer duration of diabetes or poor glycemic control were reported to be associated with the low muscle quality. ${ }^{12}$ Given these findings, sarcopenia and low muscle quality may be considered as one of the chronic complications related to diabetes.

Several recent studies have reported that the association was between diabetic microvascular complications and sarcopenia. Andersen et al demonstrated that both diabetic neuropathy (DN) and DR are significantly associated with muscle weakness of lower extremities (ankle and knee) in the univariate models and the statistical significance of DN but not DR remained unchanged in the multivariate models. ${ }^{13}$ Andreassen et al reported that DN could be involved in the accelerated muscle atrophy of lower extremities, but no difference was observed for the muscle quality between neuropathic and non-neuropathic patients. ${ }^{14}$ These findings suggest that DN may have an adverse effect on mobility through sarcopenia in patients with diabetes. Also, DR may be a cause for mobility limitations, and the association between visual impairment and sarcopenia has been reported. ${ }^{15}$ However, it remains unknown the association between the severity of DR and sarcopenia in patients with diabetes. In addition, no study has investigated the association of DR with muscle quality so far. We therefore examined whether the existence and the severity of DR could be associated with the prevalent sarcopenia and muscle quality in patients with type 2 diabetes.

\section{MATERIALS AND METHODS}

\section{Subject}

Patients with type 2 diabetes who regularly visited to the outpatient clinic at Tokyo Medical and Dental University Hospital participated in this study. Patients were eligible, if they were aged 20 years or older, and patients who had been taken a funduscopy, measured a grip strength, and undergone the whole body dual-energy X-ray absorptiometry (DXA) between 1 July 2012 and 31 October 2016 were enrolled. Patients with severe renal impairment (estimated glomerular filtration rate (eGFR) $<15 \mathrm{~mL} /$ $\min / 1.73 \mathrm{~m}^{2}$ or undergoing renal replacement therapy), pregnant women, and those with infectious or malignant diseases were excluded. Type 2 diabetes was diagnosed according to the criteria of the Japan Diabetes Society (JDS). ${ }^{16}$ This study complies with the principles laid by the Declaration of Helsinki and has been approved by the ethical committee of Tokyo Medical and Dental University (M2016-220).
Clinical and biochemical analysis

Standardized questionnaires were used to obtain information on medication and medical history. HbAlc was measured using the latex agglutination method. HbAlc levels were expressed in accordance with the National Glycohemoglobin Standardization Programs recommended by the JDS. ${ }^{16}$ The GFR was estimated using the following equation for the Japanese, as proposed by the Japanese Society of Nephrology ${ }^{17}$ : GFR $=194 \times \mathrm{SCr}^{-1.094} \times$ age $^{-0.287}$ ( (if female) $\times 0.739$ ), where SCr stands for serum creatinine in $\mathrm{mg} / \mathrm{dL}$, measured by an enzymatic method. Urinary albumin and creatinine excretion were measured by the turbidimetric immunoassay and enzymatic method, in a spot urine collection. Urinary albumin-to-creatinine ratio (ACR) was used for the assessment of albuminuria. The body mass index (BMI) was calculated as the weight divided by the square of height $\left(\mathrm{kg} / \mathrm{m}^{2}\right)$. Regional fat and lean mass including android and gynoid were measured by the whole body DXA (Lunar iDXA, GE Healthcare, Madison, Wisconsin, USA), as described previously. ${ }^{18}$ Patients were positioned for whole-body scans in accordance with the manufacturer's protocol. The whole-body fat and fat-free mass was divided into several regions, such as arms, legs, and the trunk. Appendicular lean mass was estimated as the sum of fat-free mass of the two upper limbs and two lower limbs. The skeletal muscle index (SMI) was calculated as the appendicular lean mass divided by the square of the height $\left(\mathrm{kg} / \mathrm{m}^{2}\right)$. The average of bilateral grip strength $(\mathrm{kg})$ measured by a hand dynamometer Grip-D (TKK5401, Takei, Niigata, Japan) was used for the assessment of muscle strength. In accordance with the criteria for Asians proposed by consensus report of the Asian Working Group for Sarcopenia (AWGS), ${ }^{7}$ a low muscle strength was defined as a hand grip strength $<26.0 \mathrm{~kg}$ in male and $<18.0 \mathrm{~kg}$ female, and a low muscle mass was defined as an SMI of $<7.0$ in male and $<5.4$ in female. Patients with both low muscle strength and low muscle mass were diagnosed as having a sarcopenia. The muscle quality was defined as the muscle strength per unit of the muscle mass. The ratio of grip strength to SMI $\left(\mathrm{kg} / \mathrm{kg} / \mathrm{m}^{2}\right)$ was used to assess the muscle quality in this study.

\section{Assessment of DR}

The presence and severity of $\mathrm{DR}$ were determined by qualified ophthalmologists in our hospital. The patients were grouped into three groups: patients without diabetic retinopathy (NDR), patients with non-proliferative diabetic retinopathy (NPDR), and patients with proliferative diabetic retinopathy (PDR), according to the medical record. The information on retinopathy obtained at the nearest date to DXA examination was used.

\section{Statistical analysis}

Statistical analysis was performed using SPSS V.21.0 statistical package. Data were presented as either mean $\pm \mathrm{SD}$, median with IQR or percentages as appropriate according to data distribution. Comparisons among the groups 
were analyzed by either one-way analysis of variance (continuous variables) or the $\chi^{2}$ test (categorical variables). Logistic regression analyses were carried out to assess the cross-sectional association of the severity of DR with sarcopenia. The OR with $95 \%$ CI was calculated in the logistic regression models. Next, both univariate and multivariate linear regression analyses were performed to determine the associations between DR and muscle quality. The selection of covariates in the multivariate logistic and linear regression analyses was done by a stepwise procedure. Differences were considered to be statistically significant at $p$ value less than 0.05 .

\section{RESULTS}

\section{Characteristics of study participants}

A total of 316 Japanese patients with type 2 diabetes (mean age $65 \pm 12$ years; $38 \%$ female) were enrolled in this study. As shown in table 1, 38 and 17 patients were diagnosed as having NPDR and PDR, respectively. Patients with PDR were significantly older, had a lower grip strength, longer duration of diabetes and higher level of logarithmic-transformed ACR and were more frequently receiving insulin therapy and angiotensin receptor blockers (ARBs) than those with either NDR or NPDR. In addition, there was a significant difference in the frequency of receiving calcium channel blockers among the three groups.

\section{Association of DR with sarcopenia and its components}

As shown in figure 1, the prevalence of sarcopenia significantly increased along with the progression of DR. When comparing the association between components of sarcopenia and severity of DR, the prevalence of low muscle strength significantly increased along with the progression of DR. In contrast, no significant difference in the prevalence of low muscle mass was observed among the three groups. PDR was significantly associated with the prevalent sarcopenia (OR 3.58, 95\% CI 1.19 to 10.72, $\mathrm{p}=0.023$ ) in a univariate model (table 2). After adjustment for other covariates including age, gender, BMI, body fat and the use of ARBs selected by a stepwise regression, the association between PDR and sarcopenia remained significant (OR 7.78, 95\% CI 1.52 to 39.81, $\mathrm{p}=0.014$ ). In addition to PDR, age (OR 1.12, 95\% CI 1.06 to $1.18, \mathrm{p}<0.001$ ) was positively associated with sarcopenia and BMI (OR 0.67, 95\% CI 0.55 to $0.81, \mathrm{p}<0.001$ ) and the use of ARBs (OR $0.25,95 \%$ CI 0.09 to $0.69, \mathrm{p}=0.007$ ) were negatively associated with the risk for prevalent sarcopenia. The similar findings were observed in the association of PDR with a low muscle strength; ORs of PDR for the prevalence of low muscle strength were 14.6 (95\% CI 3.17 to $67.21, \mathrm{p}<0.001)$ in a univariate and 6.25 (95\% CI 1.15 to $33.96, \mathrm{p}=0.034$ ) in a multivariate model. Other selected covariates were age (OR 1.09, 95\% CI 1.06 to $1.13, \mathrm{p}<0.001)$, the use of insulin (OR 2.14, 95\% CI 1.06 to $4.33, \mathrm{p}=0.034)$, history of cardiovascular disease (CVD) (OR 2.22, 95\% CI 0.93 to 5.29, $\mathrm{p}=0.073$ ) and male sex (OR $0.28,95 \%$ CI 0.15 to $0.55, \mathrm{p}<0.001)$. In contrast,
PDR was not significantly associated with a low muscle mass in both the univariate and multivariate models (table 2). Age (OR 1.06, 95\% CI 1.02 to $1.11, \mathrm{p}=0.004$ ), male sex (OR 6.87, 95\% CI 2.73 to $15.03, \mathrm{p}<0.001$ ) and body fat (OR $1.26,95 \%$ CI 1.14 to $1.39, \mathrm{p}<0.001$ ) were positively associated with a low muscle mass and BMI (OR $0.41,95 \% \mathrm{CI} 0.31$ to $0.54, \mathrm{p}<0.001)$ and the use of ARBs (OR $0.41,95 \%$ CI 0.17 to $0.98, \mathrm{p}=0.044$ ) were negatively associated with a low muscle mass.

\section{Association between DR and muscle quality}

Figure 2 showed that the muscle quality was lower in patients with PDR than that in patients with either NDR or NPDR. In the univariate linear regression model (table 3), the existence of DR was significantly associated with a low muscle quality (standardised $\beta-0.189$, $\mathrm{p}=0.002$ for NPDR, standardised $\beta-0.235, \mathrm{p}<0.001$ for PDR). The existence of DR remained to be significantly associated with a low muscle quality after adjustment for covariates including age and gender (standardised $\beta-0.141, p=0.006$ for NPDR, standardised $\beta-0.145$, $\mathrm{p}=0.005$ for PDR). Further adjustment for body fat and history of CVD did not attenuate the significant association between DR and the muscle quality (standardised $\beta-0.136, p=0.005$ for NPDR, standardised $\beta-0.146$, $\mathrm{p}=0.003$ for $\mathrm{PDR}$ ).

\section{DISCUSSIONS}

In the present study, we demonstrated for the first time that type 2 diabetic patients with DR, especially those with PDR, are at a high risk for the prevalent sarcopenia and the increased risk of sarcopenia in patients with DR could be attributable mainly to the impairment of muscle strength. Furthermore, we also revealed that the existence of DR is significantly associated with a low muscle quality in patients with type 2 diabetes.

Although sarcopenia is diagnosed with both low muscle mass and strength, ${ }^{6} 7$ several studies have documented that a low muscle strength is more strongly associated with both physical disability and mobility limitations than a low muscle mass. ${ }^{19}{ }^{20}$ Regarding the muscle strength, it is well established that the muscle weakness of lower extremities is associated with mobility limitations ${ }^{21}{ }^{22}$ and a low handgrip strength is also associated with the muscle weakness of lower extremities, muscle strength, and mobility limitations. ${ }^{23}$ Moreover, recent community-based studies revealed that a low handgrip strength was a significant predictor of CVD and mortality. ${ }^{24}{ }^{25}$ In our data, PDR was significantly associated with a low muscle strength in the multivariate logistic regression analyses (table 2), suggesting that the association of PDR with incident CVD and mortality in patients with diabetes might be partly explained by the coexistence of sarcopenia.

The reduction of muscle strength with aging can be partly explained by the parallel decline in muscle mass. ${ }^{26}$ However, the reduction of muscle strength with aging occurred more rapidly than that is expected by the 
Table 1 Clinical characteristics and medications according to the stages of retinopathy

\begin{tabular}{|c|c|c|c|c|}
\hline & NDR $(n=261)$ & NPDR $(n=38)$ & PDR $(n=17)$ & p Value \\
\hline Age (years) & $63 \pm 12$ & $66 \pm 12$ & $71 \pm 12$ & $<0.001$ \\
\hline Gender (\%male) & 63 & 61 & 54 & 0.236 \\
\hline Body mass index $\left(\mathrm{kg} / \mathrm{m}^{2}\right)$ & $24.8 \pm 4.4$ & $25.6 \pm 4.1$ & $24.3 \pm 3.3$ & 0.534 \\
\hline Grip strength (kg) & $27.8 \pm 9.7$ & $22.9 \pm 9.7$ & $17.7 \pm 5.7$ & $<0.001$ \\
\hline \multicolumn{5}{|l|}{ Fat free mass } \\
\hline Upper extremities (kg) & $4.5 \pm 1.3$ & $4.3 \pm 1.3$ & $3.6 \pm 1.3$ & 0.025 \\
\hline Lower extremities $(\mathrm{kg})$ & $13.4 \pm 3.4$ & $13.7 \pm 3.9$ & $11.9 \pm 3.6$ & 0.217 \\
\hline Skeletal muscle index & $6.7 \pm 1.2$ & $6.8 \pm 1.3$ & $6.3 \pm 1.2$ & 0.412 \\
\hline Andoroid (kg) & $2.1 \pm 1.1$ & $2.3 \pm 1.3$ & $2.2 \pm 1.0$ & 0.423 \\
\hline Gynoid (kg) & $3.0 \pm 1.2$ & $3.4 \pm 1.7$ & $2.9 \pm 0.8$ & 0.225 \\
\hline $\mathrm{A} / \mathrm{G}$ ratio & $0.68 \pm 0.20$ & $0.67 \pm 0.20$ & $0.73 \pm 0.24$ & 0.587 \\
\hline Body fat (\%) & $33.6 \pm 8.0$ & $34.4 \pm 8.8$ & $36.6 \pm 5.7$ & 0.361 \\
\hline $\begin{array}{l}\text { Duration of diabetes } \\
\text { (years) }\end{array}$ & $6(5-7)$ & $11(8-14)$ & $23(16-30)$ & $<0.001$ \\
\hline History of CVD (\%) & 10.3 & 13.5 & 6.7 & 0.739 \\
\hline $\mathrm{SBP}(\mathrm{mm} \mathrm{Hg})$ & $127 \pm 15$ & $128 \pm 12$ & $140 \pm 15$ & 0.001 \\
\hline $\mathrm{DBP}(\mathrm{mm} \mathrm{Hg})$ & $75 \pm 13$ & $73 \pm 11$ & $77 \pm 18$ & 0.499 \\
\hline $\log$ ACR (mg/g) & $1.47 \pm 0.55$ & $1.63 \pm 0.64$ & $2.30 \pm 0.74$ & $<0.001$ \\
\hline eGFR $\left(\mathrm{mL} / \mathrm{min} / 1.73 \mathrm{~m}^{2}\right)$ & $74.2 \pm 19.4$ & $70.1 \pm 29.7$ & $64.6 \pm 30.3$ & 0.151 \\
\hline HbA1c (\%) & $7.2 \pm 1.4$ & $7.4 \pm 1.7$ & $7.7 \pm 1.0$ & 0.266 \\
\hline $\mathrm{HbA} 1 \mathrm{c}(\mathrm{mmol} / \mathrm{mol})$ & $55 \pm 8$ & $57 \pm 12$ & $60 \pm 5$ & \\
\hline Triglycerides (mmol/L) & $1.70(1.56-1.85)$ & $1.70(1.18-2.22)$ & $1.69(1.19-2.20)$ & 0.999 \\
\hline HDL cholesterol (mmol/L) & $1.54 \pm 0.47$ & $1.46 \pm 0.40$ & $1.47 \pm 0.34$ & 0.563 \\
\hline LDL cholesterol (mmol/L) & $2.94 \pm 0.38$ & $2.84 \pm 0.69$ & $2.89 \pm 0.95$ & 0.889 \\
\hline $\mathrm{UA}(\mu \mathrm{mol} / \mathrm{L})$ & $320 \pm 80$ & $314 \pm 73$ & $332 \pm 68$ & 0.752 \\
\hline Insulin (\%) & 24 & 32 & 100 & $<0.001$ \\
\hline Sulfonylureas (\%) & 28 & 27 & 14 & 0.741 \\
\hline Metoformins (\%) & 48 & 46 & 47 & 0.947 \\
\hline Alpha-Gls (\%) & 9 & 12 & 14 & 0.865 \\
\hline Glinides (\%) & 8 & 6 & 0 & 0.733 \\
\hline TZDs (\%) & 10 & 9 & 0 & 0.687 \\
\hline DPP4 inhibitors (\%) & 58 & 73 & 43 & 0.195 \\
\hline SGLT2 inhibitors (\%) & 3 & 0 & 0 & 0.543 \\
\hline GLP1-RAs (\%) & 2 & 4 & 0 & 0.464 \\
\hline ARBs (\%) & 30 & 58 & 71 & $<0.001$ \\
\hline CCBs (\%) & 30 & 26 & 88 & $<0.001$ \\
\hline Diuretics (\%) & 8 & 5 & 18 & 0.339 \\
\hline Statins (\%) & 30 & 40 & 53 & 0.076 \\
\hline UA lowering agents (\%) & 3 & 7 & 5 & 0.415 \\
\hline Antiplatelet agents (\%) & 13 & 13 & 18 & 0.837 \\
\hline
\end{tabular}

ACR, albumin-to-creatinine ratio; A/G, android-to-gynoid; ARBs, angiotensin receptor blockers; CCBs, calcium channel blockers; CVD, cardiovascular disease; DBP, diastolic blood pressure; DPP4, dipeptidyl peptidase 4; eGFR, estimated glomerular filtration ratio; Gls, glycosidase inhibitors; GLP1-RA, glucagon-like peptide-1 receptors agonist; HDL, high-density lipoprotein; LDL, low-density lipoprotein; NDR, no diabetic retinopathy; NPDR, non-proliferative diabetic retinopathy; PDR proliferative diabetic retinopathy; SBP, systolic blood pressure; SGLT2, sodium-glucose cotransporter 2; TZDs, thiazolidinediones; UA, uric acid. 


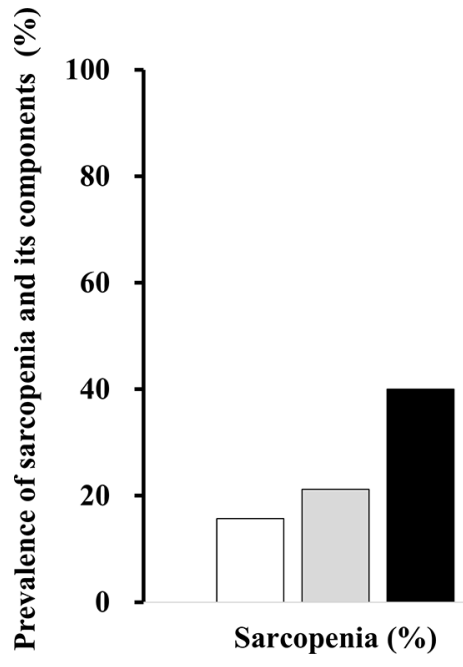

$\square$ NDR $\square$ NPDR $\square$ PDR

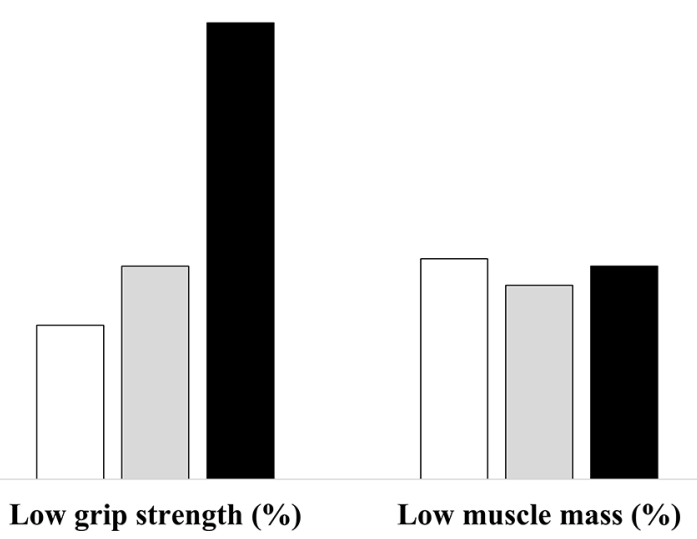

Figure 1 Difference in the prevalence of sarcopenia and its components (low muscle strength and low muscle mass) between NDR, NPDR and PDR. NDR, no diabetic retinopathy; NPDR, non-proliferative diabetic retinopathy; PDR, proliferative diabetic retinopathy.

decline in muscle mass, and the contradiction between muscle mass and strength was thought to be caused by a decline in muscle quality. ${ }^{20}$ In a 12 -year longitudinal study of a community-dwelling population in Japan, the age-related decrease in muscle mass was relatively small, but the muscle quality significantly decreased with aging. ${ }^{27}$ Other than aging, the Health ABC study reported that the decline in muscle quality was accelerated by the presence of diabetes. ${ }^{10}$ Another recent study demonstrated that diabetic patients with a poor glycemic control $(\mathrm{HbA} 1 \mathrm{c} \geq 8.5 \%)$ were at a significantly increased risk for the decline in muscle quality and the impaired functional ability (assessed using Short Physical Performance Battery) compared with either patients with a HbA1c of $<8.5 \%$ or subjects without diabetes. ${ }^{28}$ Those studies implied that a muscle quality plays a key role in the relationship between diabetes and mobility limitations. We revealed for the first time in this study that DR is strongly associated with a low muscle quality (table 3 ), presumably suggesting that the screening of DR would be useful to estimate the risks for the low muscle quality in patients with diabetes.

Among diabetic patients, some investigators have recently focused on the association of diabetic

Table 2 ORs of sarcopenia, low muscle strength and low muscle mass in patients with type 2 diabetes

\begin{tabular}{|c|c|c|c|c|c|c|c|c|c|}
\hline & \multicolumn{3}{|c|}{$\begin{array}{l}\text { Sarcopenia low muscle } \\
\text { strength+low muscle mass }\end{array}$} & \multicolumn{3}{|c|}{$\begin{array}{l}\text { Low muscle strength } \\
\text { Grip strength }<26 \mathrm{~kg} \text { in male } \\
\text { Grip strength }<18 \mathrm{~kg} \text { in female }\end{array}$} & \multicolumn{2}{|c|}{$\begin{array}{l}\text { Low muscle mass } \\
\mathrm{SMI}<7.0 \text { in male } \\
\mathrm{SMI}<5.4 \text { in female }\end{array}$} & \multirow[b]{2}{*}{$p$ Value } \\
\hline & OR & $(95 \% \mathrm{Cl})$ & $p$ Value & OR & $(95 \% \mathrm{Cl})$ & p Value & OR & $(95 \% \mathrm{Cl})$ & \\
\hline \multicolumn{10}{|l|}{ Univariate model } \\
\hline NPDR & 1.50 & (0.60 to 3.76$)$ & 0.385 & 1.78 & (0.77 to 3.81$)$ & 0.184 & 0.74 & (0.34 to 1.62$)$ & 0.452 \\
\hline PDR & 3.58 & (1.19 to 10.72 ) & 0.023 & 14.60 & (3.17 to 67.21 ) & $<0.001$ & 0.94 & (0.38 to 2.75 ) & 0.915 \\
\hline \multicolumn{10}{|l|}{ Multivariate model } \\
\hline NPDR & 3.79 & (1.14 to 12.65$)$ & 0.030 & 1.08 & (0.43 to 2.71 ) & 0.878 & 2.49 & (0.76 to 8.10$)$ & 0.130 \\
\hline PDR & 7.78 & (1.52 to 39.81 ) & 0.014 & 6.25 & (1.15 to 33.96$)$ & 0.034 & 2.34 & (0.35 to 15.71$)$ & 0.383 \\
\hline Age (years) & 1.12 & (1.06 to 1.18 ) & $<0.001$ & 1.09 & (1.06 to 1.13 ) & $<0.001$ & 1.06 & (1.02 to 1.11$)$ & 0.004 \\
\hline $\begin{array}{l}\text { Gender (male vs } \\
\text { female) }\end{array}$ & 7.26 & (2.11 to 24.97 ) & 0.002 & 0.28 & (0.15 to 0.55$)$ & $<0.001$ & 6.87 & (2.73 to 15.03 ) & $<0.001$ \\
\hline $\mathrm{BMI}\left(\mathrm{kg} / \mathrm{m}^{2}\right)$ & 0.67 & (0.55 to 0.81 ) & $<0.001$ & NA & & & 0.41 & (0.31 to 0.54 ) & $<0.001$ \\
\hline Body fat (\%) & 1.16 & (1.07 to 1.27 ) & 0.001 & NA & & & 1.26 & (1.14 to 1.39$)$ & $<0.001$ \\
\hline ARBs & 0.25 & (0.09 to 0.69 ) & 0.007 & NA & & & 0.41 & (0.17 to 0.98$)$ & 0.044 \\
\hline Insulin & NA & & & 2.14 & (1.06 to 4.33$)$ & 0.034 & NA & & \\
\hline History of CVD & NA & & & 2.22 & (0.93 to 5.29$)$ & 0.073 & NA & & \\
\hline
\end{tabular}

ARBs, angiotensin receptor blockers; BMI, body mass index; CVD, cardiovascular disease; NPDR, non-proliferative diabetic retinopathy; PDR, proliferative diabetic retinopathy; SMI, skeletal muscle index. 


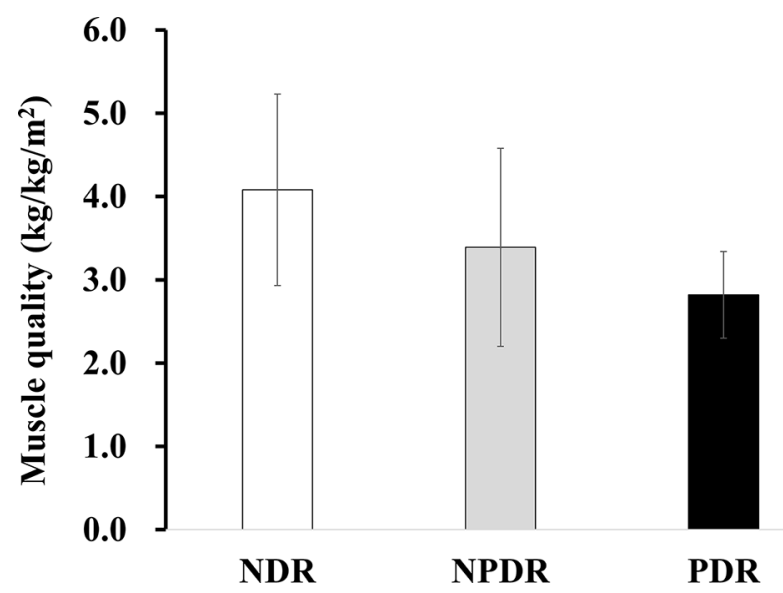

Figure 2 Muscle quality (mean \pm SE, \%) of the patients with NDR, NPDR and PDR. NDR, no diabetic retinopathy; NPDR, non-proliferative diabetic retinopathy; PDR, proliferative diabetic retinopathy.

microvascular complications with a muscle mass, strength, and quality. In a cross-sectional study comprising 36 patients with type 2 diabetes and 36 control subjects, Andersen et al reported that the existence of both $\mathrm{DN}$ and DR was associated with a muscle weakness of lower extremities in a univariate analysis. ${ }^{13}$ In a multivariate analysis, there was a significant association between DN and a muscle weakness of lower extremities, whereas the

Table 3 Association between diabetic retinopathy and muscle quality in patients with type 2 diabetes

\begin{tabular}{lll}
\hline & Standardised $\beta$ & p Values \\
\hline $\begin{array}{l}\text { Univariate model (adjusted } \\
\left.\mathrm{R}^{2}=0.08\right)\end{array}$ & & \\
\hline NPDR & -0.189 & 0.002 \\
\hline PDR & -0.235 & $<0.001$ \\
\hline Age-adjusted and gender- & & \\
adjusted model (adjusted & & \\
$\left.\mathrm{R}^{2}=0.37\right)$ & & \\
\hline NPDR & -0.141 & 0.006 \\
\hline PDR & -0.145 & 0.005 \\
\hline Age & -0.210 & $<0.001$ \\
\hline Gender & 0.496 & $<0.001$ \\
\hline Multivariate model (adjusted & & \\
$\left.R^{2}=0.46\right)$ & & \\
\hline NPDR & -0.136 & 0.005 \\
\hline PDR & -0.146 & 0.003 \\
\hline Age & -0.238 & $<0001$ \\
\hline Gender & 0.363 & $<0.001$ \\
\hline Body fat & -0.315 & $<0.001$ \\
\hline History of CVD & -0.141 & 0.004 \\
\hline
\end{tabular}

Muscle quality was defined as grip strength divided by skeletal muscle index.

CVD, cardiovascular disease; NPDR, non-proliferative diabetic retinopathy; PDR, proliferative diabetic retinopathy. statistical significance of DR disappeared. It is assumed that the impact of DN on the muscle strength of lower extremities may be stronger than that of DR; however, no information was available regarding the association between muscle mass and the severity of DR. In addition, the sample size of the study was small; therefore, concluding insufficient evidence for whether DR could be associated with a sarcopenia defined as both low muscle mass and strength. In this regard, we believe that our data in this study is the first to show evidence for the clinical relevance of DR with both sarcopenia and muscle quality in patients with type 2 diabetes.

As shown in table 2, we revealed apparent differences in the impact of gender on a low muscle strength and low muscle mass (male were protective against the decline of grip strength as were female against the low muscle mass). Both low muscle strength and low muscle mass were defined by the criteria for Asians, proposed by consensus report of the AWGS. ${ }^{7}$ However, the cut-off values were established on the basis of the data obtained mainly from subjects without diabetes, and it has still been unclear whether the criteria could be applied for patients with diabetes. Further studies may be needed to identify cut-off values for the diagnosis of sarcopenia in patients with diabetes.

We also found a significant association of ARBs with sarcopenia in patients with diabetes (table 2). Previous reports showed the potential of renin angiotensin aldosterone system inhibitors to protect against the decline of muscle strength. ${ }^{29}{ }^{30}$ Onder et al reported that ACE inhibitors can prevent the decline in muscle strength in elderly women with hypertension in a 5-year longitudinal observational study. ${ }^{29}$ In the cross-sectional survey of 2431 participants from the Health ABC Study, the use of ACE inhibitors was significantly associated with a larger muscle mass in lower extremities. ${ }^{30}$ Our data are consistent with the results of these previous studies ${ }^{29} 30$ and suggest the possibility that ARBs may prevent the progression of sarcopenia in patients with diabetes.

This study has several limitations. First, it is impossible to infer causality because of its cross-sectional design, although we revealed the significant association between DR and sarcopenia in patients with type 2 diabetes. Second, the characteristics of subjects in this study were homogenous; therefore, the generalization of our findings might be limited. Third, information on diet, exercise and visual acuity that could affect both muscle mass and strength was unavailable. Visual impairment may lead to lack of exercise, resulting in the progression of sarcopenia or the decline in muscle quality. Finally, we were unable to obtain information on DN. DN was recently reported to be strongly associated with a low muscle strength, low muscle mass and decline in muscle quality. ${ }^{13} 1431$ Therefore, further studies are needed in order to reveal the association of both DR and DN with sarcopenia and/or muscle quality.

In conclusion, this is the first study to investigate the association of DR, especially PDR with sarcopenia, which 
is defined as both low muscle mass and strength. Our data further suggest that type 2 diabetic patients with either NPDR or PDR are at a high risk for the low muscle quality compared with patients with NDR.

Acknowledgments The authors would like to thank all the staff of the Department of Molecular Endocrinology and Metabolism, Tokyo Medical and Dental University for their contributions.

Contributors RB and TF designed the study, researched data, and wrote and edited the manuscript. RB, IM, TY, and YO contributed to intellectual discussion and reviewed and edited the manuscript. TT, YN, MM, HI, and KH researched data. As the corresponding author and guarantor of this manuscript, $\mathrm{RB}$ is the guarantor of this work and, as such, had full access to all the data in the study and takes responsibility for the integrity of the data and the accuracy of the data analysis.

Funding This work was partially supported by Grants-in-Aid for Scientific Research from the Ministry of Education, Culture, Sports, Science and Technology of Japan, Grants-in-Aid from the Ministry of Health, Labor, and Welfare of Japan (Comprehensive Research on Lifestyle-Related Diseases Including Cardiovascular Diseases and Diabetes Mellitus) and Grants-in-Aid from Astellas Foundation for Research on Metabolic Disorders.

\section{Competing interests None declared.}

Ethics approval The ethical committee of Tokyo Medical and Dental University (M2016-220).

Provenance and peer review Not commissioned; externally peer reviewed. Data sharing statement № additional data are available.

Open Access This is an Open Access article distributed in accordance with the Creative Commons Attribution Non Commercial (CC BY-NC 4.0) license, which permits others to distribute, remix, adapt, build upon this work non-commercially, and license their derivative works on different terms, provided the original work is properly cited and the use is non-commercial. See: http://creativecommons.org/ licenses/by-nc/4.0/

(c) Article author(s) (or their employer(s) unless otherwise stated in the text of the article) 2017. All rights reserved. No commercial use is permitted unless otherwise expressly granted.

\section{REFERENCES}

1. Bunce $C$, Wormald R. Leading causes of certification for blindness and partial sight in England \& Wales. BMC Public Health 2006:6:58.

2. Yau JW, Rogers SL, Kawasaki R, et al. Global prevalence and major risk factors of diabetic retinopathy. Diabetes Care 2012;35:556-64.

3. Cheung N, Mitchell P, Wong TY. Diabetic retinopathy. Lancet 2010;376:124-36.

4. Fong DS, Aiello LP, Ferris FL, et al. Diabetic retinopathy. Diabetes Care 2004;27:2540-53.

5. Kramer CK, Rodrigues TC, Canani LH, et al. Diabetic retinopathy predicts all-cause mortality and cardiovascular events in both type 1 and 2 diabetes: meta-analysis of observational studies. Diabetes Care 2011;34:1238-44.

6. Cruz-Jentoft AJ, Baeyens JP, Bauer JM, et al. Sarcopenia: European consensus on definition and diagnosis: report of the European working group on sarcopenia in older people. Age Ageing 2010;39:412-23.

7. Chen LK, Liu LK, Woo J, et al. Sarcopenia in Asia: consensus report of the Asian working group for sarcopenia. J Am Med Dir Assoc 2014;15:95-101.

8. Kalyani RR, Corriere M, Ferrucci L. Age-related and disease-related muscle loss: the effect of diabetes, obesity, and other diseases. Lancet Diabetes Endocrinol 2014;2:819-29.

9. Bianchi L, Volpato S. Muscle dysfunction in type 2 diabetes: a major threat to patient's mobility and independence. Acta Diabetol 2016;53:879-89.
10. Park SW, Goodpaster BH, Strotmeyer ES, et al. Accelerated loss of skeletal muscle strength in older adults with type 2 diabetes: the health, aging, and body composition study. Diabetes Care 2007;30:1507-12.

11. Volpato S, Bianchi L, Lauretani F, et al. Role of muscle mass and muscle quality in the association between diabetes and gait speed. Diabetes Care 2012;35:1672-9.

12. Park SW, Goodpaster BH, Strotmeyer ES, et al. Decreased muscle strength and quality in older adults with type 2 diabetes: the health, aging, and body composition study. Diabetes 2006;55:1813-8.

13. Andersen $\mathrm{H}$, Nielsen $\mathrm{S}$, Mogensen CE, et al. Muscle strength in type 2 diabetes. Diabetes 2004;53:1543-8.

14. Andreassen CS, Jakobsen J, Ringgaard S, et al. Accelerated atrophy of lower leg and foot muscles--a follow-up study of long-term diabetic polyneuropathy using magnetic resonance imaging (MRI). Diabetologia 2009;52:1182-91.

15. Landi F, Liperoti R, Russo A, et al. Sarcopenia as a risk factor for falls in elderly individuals: results from the ilSIRENTE study. Clin Nutr 2012;31:652-8

16. Seino Y, Nanjo K, Tajima N, et al. Report of the committee on the classification and diagnostic criteria of diabetes mellitus. J Diabetes Investig 2010;1:212-28.

17. Matsuo S, Imai E, Horio M, et al. Revised equations for estimated GFR from serum creatinine in Japan. Am J Kidney Dis 2009;53:982-92.

18. Kang SM, Yoon JW, Ahn HY, et al. Android fat depot is more closely associated with metabolic syndrome than abdominal visceral fat in elderly people. PLoS One 2011;6:e27694.

19. Rantanen T, Guralnik JM, Foley D, et al. Midlife hand grip strength as a predictor of old age disability. JAMA 1999;281:558-60.

20. Goodpaster BH, Park SW, Harris TB, et al. The loss of skeletal muscle strength, mass, and quality in older adults: the health, aging and body composition study. J Gerontol A Biol Sci Med Sci 2006:61:1059-64.

21. Visser M, Deeg DJ, Lips P, et al. Skeletal muscle mass and muscle strength in relation to lower-extremity performance in older men and women. J Am Geriatr Soc 2000;48:381-6.

22. Visser M, Goodpaster BH, Kritchevsky SB, et al. Muscle mass, muscle strength, and muscle fat infiltration as predictors of incident mobility limitations in well-functioning older persons. $J$ Gerontol $A$ Biol Sci Med Sci 2005;60:324-33.

23. Lauretani F, Russo CR, Bandinelli S, et al. Age-associated changes in skeletal muscles and their effect on mobility: an operational diagnosis of sarcopenia. J Appl Physio/2003;95:1851-60.

24. Kishimoto H, Hata J, Ninomiya T, et al. Midlife and late-life handgrip strength and risk of cause-specific death in a general Japanese population: the Hisayama Study. J Epidemiol Community Health 2014:68:663-8.

25. Leong DP, Teo KK, Rangarajan S, et al. Prognostic value of grip strength: findings from the Prospective Urban Rural Epidemiology (PURE) study. Lancet 2015;386:266-73.

26. Stenholm S, Harris TB, Rantanen T, et al. Sarcopenic obesity: definition, cause and consequences. Curr Opin Clin Nutr Metab Care 2008;11:693-700.

27. Shimokata $\mathrm{H}$, Ando $\mathrm{F}$, Yuki $\mathrm{A}$, et al. Age-related changes in skeletal muscle mass among community-dwelling Japanese: a 12-year longitudinal study. Geriatr Gerontol Int 2014;14(Suppl 1):85-92.

28. Yoon JW, Ha YC, Kim KM, et al. Hyperglycemia is associated with impaired muscle quality in older men with diabetes: the Korean longitudinal study on health and aging. Diabetes Metab $J$ 2016:40:140-6.

29. Onder G, Penninx BW, Balkrishnan R, et al. Relation between use of angiotensin-converting enzyme inhibitors and muscle strength and physical function in older women: an observational study. Lancet 2002;359:926-30.

30. Di Bari M, van de Poll-Franse LV, Onder G, et al. Antihypertensive medications and differences in muscle mass in older persons: the Health, Aging and Body Composition Study. J Am Geriatr Soc 2004:52:961-6.

31. Moore CW, Allen MD, Kimpinski K, et al. Reduced skeletal muscle quantity and quality in patients with diabetic polyneuropathy assessed by magnetic resonance imaging. Muscle Nerve 2016;53:726-32. 